\title{
Biomimetic Spider-web like Electrospun Nanofibrous Membrane of Nylon-6 for Future Air Filtration
}

\author{
Hem Raj Pant \\ Department of Engineering Science and Humanities, Institute of Engineering \\ Central Campus, Pulchowk, Tribhuvan University, Nepal \\ Corresponding author: hempant2002@yahoo.com
}

\begin{abstract}
In this work, methoxy poly(ethylene glycol) (MPEG) oligomer with viscous supporting nylon-6 solution was fabricated in the form of natural spider-web like structure via an electrospinning process. Field-emission scanning electron microscopy (FE-SEM) showed that this network consists of thinner MPEG nanofibers, approximately $15 \pm 5 \mathrm{~nm}$ in diameter connected with thicker nylon-6 main nanofibers, arranged in spider-web like structure. TGA and XRD analysis showed that the hybrid mat of MPEG and nylon-6 was formed during electrospinning. These two different interconnected nanofibers were separated by extraction of MPEG from the fibrous mat in cold water and identified. The molecular weight and purity of the extracted MPEG was determined by gel permeation chromatography (GPC) and Fourier transform infrared (FT-IR) analysis, respectively. The decreased average pore diameter and increased hydrophilicity of the hybrid mat, caused by spider-web like structure, can make it as a potential candidate for air filtration.
\end{abstract}

Key words: Nylon-6; MPEG; Electrospinning; Spider-web; Hybrid mat

\section{Introduction}

In the field of nanotechnology, biomimetic polymer nanofibers have been attracting significant attention due to its wide applications in various areas such as filtration [1-4], tissue engineering [5-7], manufacture of protective clothing and pharmacy [8-10]. To prepare the nanofibers, electrospinning has been used as an efficient processing method for generating nano to submicrometer fibrous structures [11]. Since the nanofibers obtained from electrospinning possess notable characteristics such as a large surface area to volume ratio, good pore interconnectivity, high porosity and the possibility to incorporate active components on a nanoscale, it can be the best candidates in many applications. The 2D as well as 3D structure of an electrospinning fibrous mat are becoming very important in academic as well as research fields. These hybrid mats have also unique physical properties than their component polymers. Therefore, the research interest is increased in the formation of polymer hybrid mats for different application. Ding et al. demonstrated poly (acrylic acid) and nylon-6, 2D and 3D structures and were able to show the interconnected spider-web-like fibers [12]. Parajuli et al. successfully synthesized poly(acrylic acid) and nylon- 6 hybrid mat by using acrylic acid monomer and found that the polymerization of monomer during electrospinning was carried out [13]. Recently, Nasser et al. obtained spider- 
web in different polymers with different inorganic salts and found that there was increased in mechanical strength as well as hydrophilic properties [14].

However, as far as our knowledge the spider-web hybrid mat of polymer and oligomer is not reported elsewhere. Since nylon polymer has many applications as an engineering material because of its strong mechanical properties such as high tensile strength and resistance properties [15-17] due to hydrogen bonding [18], we try to make somewhat new material by mixing of nylon-6 with methoxy poly(ethylene glycol)(MPEG) oligomer. In this work, we focus to make spider-web-like structure in which thinner MPEG nanofibers were connected with thicker nylon-6 fibers. In the meantime, we also focused on the further polymerization of MPEG oligomer for producing interconnected fibrous mats via electrospinning process. The electrospinning technique has been mainly used in different research work for fiber production driven by electrostatic force. However, it can be also used for polymerization and mat fabrication simultaneously [13]. The viscous solution of polymer having functional groups present on its branches or on terminal ends can react in electric field and undergoes polymerization. During electrospinning, a high voltage is applied across the viscous solution of polymer having high molecular weight. When this solution is sprayed through the syringe spinneret, a chemical reaction can possible during the Taylor cone formation at the tip of the pendant droplets [19]. It is because of high electrostatic forces between the tip and the collector. The conductivity, viscosity and surface tension are the important factors for this process. Above the critical voltage, electrostatic forces overcome the surface tension of this electrospinning solution and an electrified polymer jet is formed [20]. If this jet contains a phaseseparated polymer layers, the phases can be interconnected into the nanofibers. At this condition jet is elongated and whipped continuously by electrostatic repulsion until there is deposition on the grounded collector in the form of a randomly interwoven fibrous mat. Due to the polar nature of formic acid the conductivity of solution mixture is increased and the polymer molecules will be polarized. Therefore, it is possible to interconnect the main fiber of nylon-6 and spider net fiber of MPEG via hydrogen bonding which is responsible for increasing the mechanical strength of nylon-6.

\section{Experimental}

\subsection{Materials}

Nylon-6 (relative viscosity 2.5, grade KN20) was obtained from Kolon (South Korea) and MPEG (MW=550 g/mol) oligomer was purchased from Aldrich (USA). Acetic acid and formic acids (analytical grade, Showa, Japan) mixture was used as solvent for nylon-6. All the chemicals were used without further purification. Doubled distilled water was used for extraction of hybrid mat.

\subsection{Procedure}

\subsubsection{Preparation of an interconnected fibrous mat}

A $22 \mathrm{wt} \%$ Nylon- 6 solution was prepared by adding nylon- 6 pellets in a mixture of formic acid and acetic acid ( $4: 1$ by Wt). After stirring for 24 hours at room temperature, calculated amount of MPEG oligomer $(\mathrm{Mw}=550)$ were added separately to get $1 \mathrm{wt} \%, 2 \mathrm{wt} \%$, and $4 \mathrm{wt} \%$ MPEG in nylon- 6 and mixture were stirred for $30 \mathrm{~min}$ at room temperature. The resulted mixture solutions were subjected for electrospinning process in a syringe fitted with a pointed nozzle, which was clamped at about a $20^{\circ}$ angle relative to the horizontal axis. The positive pole copper electrode was secured inside the solution. An electric voltage was applied at $20 \mathrm{kV}$, and a rotating steel drum covered by polyethylene sheet was used as a collector of electrospun fibers, which was positioned 
$16 \mathrm{~cm}$ from the tip of the syringe. The collected nanofibers were dried at room temperature for 24 $\mathrm{h}$ and vacuum dried for further $24 \mathrm{~h}$. Then the samples were used for analysis.

\subsubsection{Extraction of Samples}

The extraction MPEG was carried out from the hybrid NPEG/nylon-6 (MPEGN) spider net mat. A piece of mat having weight $1.2 \mathrm{~g}$ was taken from a single $2 \mathrm{wt} \%$ MPEG containing mat for extraction in cooled distilled water. $250 \mathrm{ml}$ of distilled water in $500 \mathrm{ml}$ round bottom flask with this amount of mat was shaken for $60 \mathrm{~h}$ at room temperature on electric shaker. The solution having dissolved MPEG and unaffected nylon mat were separated by filtration. The filtrate solution was taken, and the solvent was evaporated by drying in an oven at $80^{\circ} \mathrm{C}$. The resulted solute was characterized by using FT-IR and GPC.

\subsection{Characterization}

The thermal property of hybrid mat was measured by thermogravimetric analyzer (Perkin-Elmer Inc., Elmer TGA 6, Waltham, MA) under a nitrogen atmosphere. Fourier transform infrared (FTIR) spectra were recorded with KBr pellets using an ABB Bomen MB100 Spectrometer (Bomen, Canada). The molecular weight of sample was measured by gel permeation chromatography (GPC) and the measurements were made via a Waters 150C (polymer Laboratories, England) using chloroform as a mobile phase at the rate of $1.0 \mathrm{~mL} / \mathrm{min}$. The crystalline structure of mat was determined by X-ray diffractometer (Rigaku, Japan) measured radiation over a Bragg angle from 10 to $80^{\circ}$. Surface morphology of hybrid mat was studied by field-emission scanning electron microscope (FESEM, Hitachi S-7400, Hitachi, Japan) after sputter coating with osmium tetra oxide. Average pore size was measured by using a porosimeter (autoporeIV9500, micromeritics, USA). The mechanical properties of the different samples were measured with a universal testing machine (AG-5000G, Shimadzu, Japan), under crosshead speed of $10 \mathrm{~mm} / \mathrm{min}$ at room temperature. The samples were prepared in the form of standard dumbbell shapes according to ASTM Standard D 638 via die cutting from the electrospun mats and tested in the machine direction. The information about wetability was measured by the water contact angle which was measured by contact angle meter (Digidrop, GBX, France).

\section{Results and discussion}

During the electrospinning process, an electrified polymeric nylon-6 solution with MPEG oligomer was extruded through a spinneret which might have increased the energy in the reacting substrate to induce a chemical reaction during the formation of a Taylor cone (11). The interaction of the two polymers was observed via thermogravimetric analysis (TGA) and a differential thermogravimetry (DTG) graph of the MPEGN (1 wt\% MPEG) and pure nylon-6 mat (Fig. 1A). A temperature correlation with maximum thermal degradation rates for MPEG at $322^{\circ} \mathrm{C}$ and $445.8^{\circ} \mathrm{C}$ for nylon- 6 were observed in MPEGN mat. The shifting of this value from $449.6^{\circ} \mathrm{C}$ in pure nylon- 6 mat to $445.8^{\circ} \mathrm{C}$ in MPEGN mat (d of Fig. 1A) for nylon- 6 indicated the interaction of two polymers to form a hybrid mat. Furthermore, from the FT-IR spectra (Fig. 1B) of the extracted MPEG from MPEGN mat and the initially used MPEG, which were very similar, we conclude that the spiderweb is formed by the interconnection of MPEG fibers with Nylon-6 fibers. The FT-IR spectra of MPEGN and extracted MPEG were compared. The peak at $3300 \mathrm{~cm}^{-1}$ was not seen in extracted MPEG which showed that the extracted sample did not contain nylon-6. Shifting of bands from 975 to $848 \mathrm{~cm}^{-1}$ and from 1741 to $1722 \mathrm{~cm}^{-1}$ for nylon-6 in MPEGN (Fig. 2) indicates the formation of stronger hydrogen bonds [8]. A new peak at $1120 \mathrm{~cm}^{-1}$ in the MPEGN- IR spectra, which was 
not observed in pure nylon-6, indicates the presence of the C-O group of MPEG. This information shows the formation of hybrid mat of two polymers.
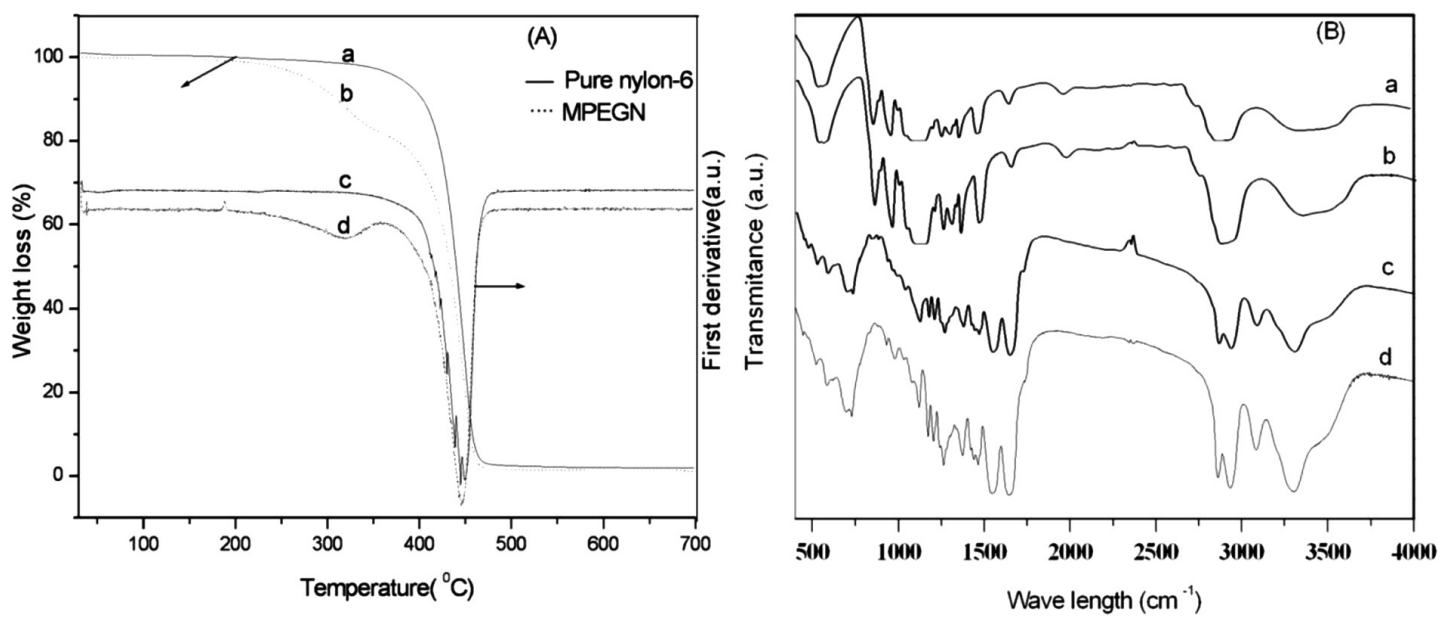

Fig. 1. (A) TGA and DTG of pure and $1 \mathrm{wt} \%$ MPEG nylon-6 mat, (B) FT-IR spectra of (a) initially used MPEG, (b) extracted MPEG, (c) MPEGN mat, and (d) pure nylon-6 mat.

In viscous nylon-6 solution, MPEG oligomer molecules can combine with each other to create higher molecular weight molecules during fabrication by electrospinning, which was supported by GPC (Fig. 2). The GPC data showed that the molecular weight of the extracted sample was about $1110 \mathrm{gm} / \mathrm{mole}$ which is about two times of initially used MPEG $(\mathrm{Mw}=550)$. If the viscous polymer solution is extruded through a spinneret during electrospinning, a polymer jet will form due to electrostatic forces. The residual charges in this jet begin to repel each other when they become separated from the electrode. The polymer layer then becomes cross-linked to form an interconnected fibrous mat. The formation of hybrid mat was further conformed by XRD patterns of pure and hybrid nylon-6 mat (Fig. 3). The shifting of main peak of nylon-6 towards lower value with broaden the peak is the indication of good interaction of MPEG with nylon-6.

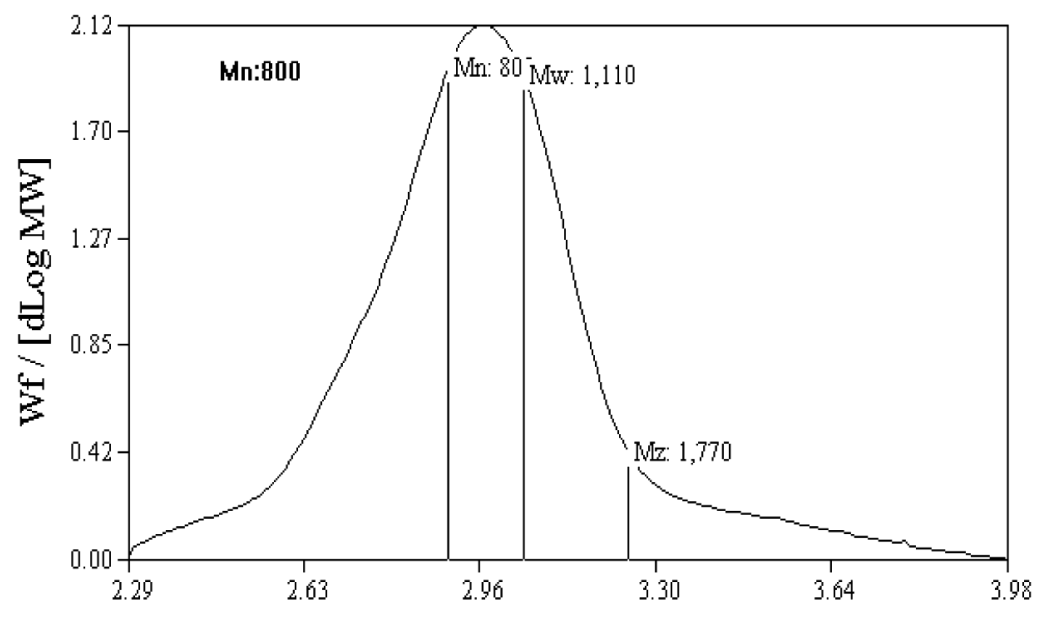

Fig. 2. GPC of extracted MPEG from MPEGN electrospun mat. 


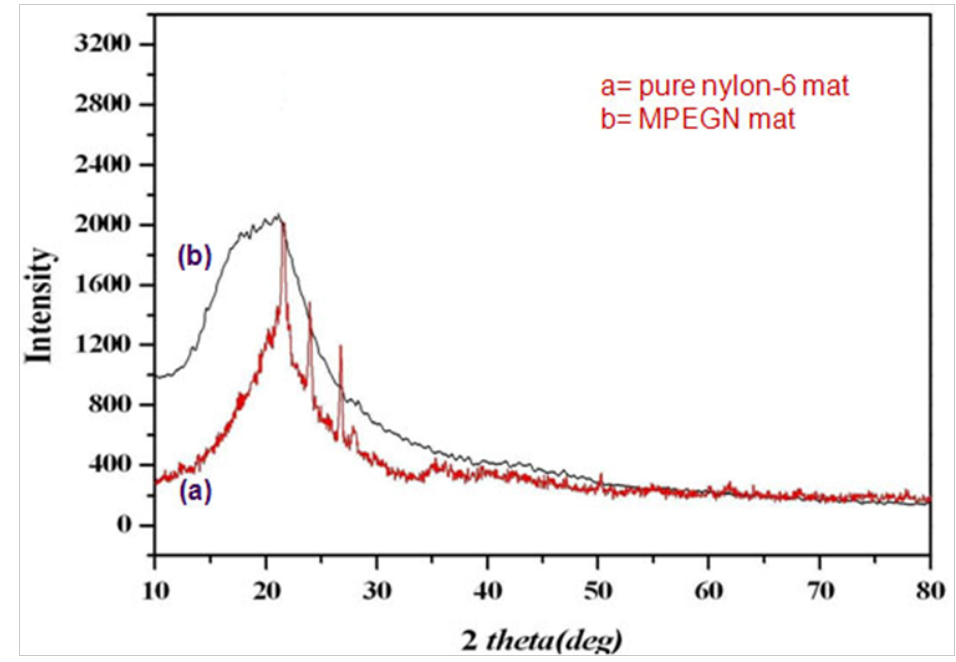

Fig. 3. XRD patterns of nylon-6 (a) and MPEGN mats (b).

The FE-SEM image of pure nylon-6 mat (Fig. 4A) and of mats with different amounts of MPEG in MPEGN (Fig. 4B and 5) showed that nylon-6 mat has no spider-web structure but that MPEGN mats have highly interconnected spider-web structures. The average fiber diameter of the thin and thick nanofibers were found to be $15 \pm 5 \mathrm{~nm}$ and $300 \pm 100 \mathrm{~nm}$, respectively. We found that the diameters of nylon- 6 nanofibers decreased with increasing MPEG content. FE-SEM images of electrospun mats before and after the extraction process are shown in Fig. 6 (A and B), respectively. This demonstrates that the interconnected fibrous MPEGN mats underwent a change in morphology (i.e. thin fibers dissolved upon exposure to water while the thick fibers remained intact). Thus, it can be confirmed that the thick nanofibers were composed of nylon- 6 and the thin ones of MPEG. Fig. 4 (B) shows that the $1 \mathrm{wt} \%$ MPEG in nylon- 6 mat has more spider-webs structure as compared to the mat with 2 and $4 \mathrm{wt} \%$ MPEG in nylon-6 mat (Fig. 5B and D).
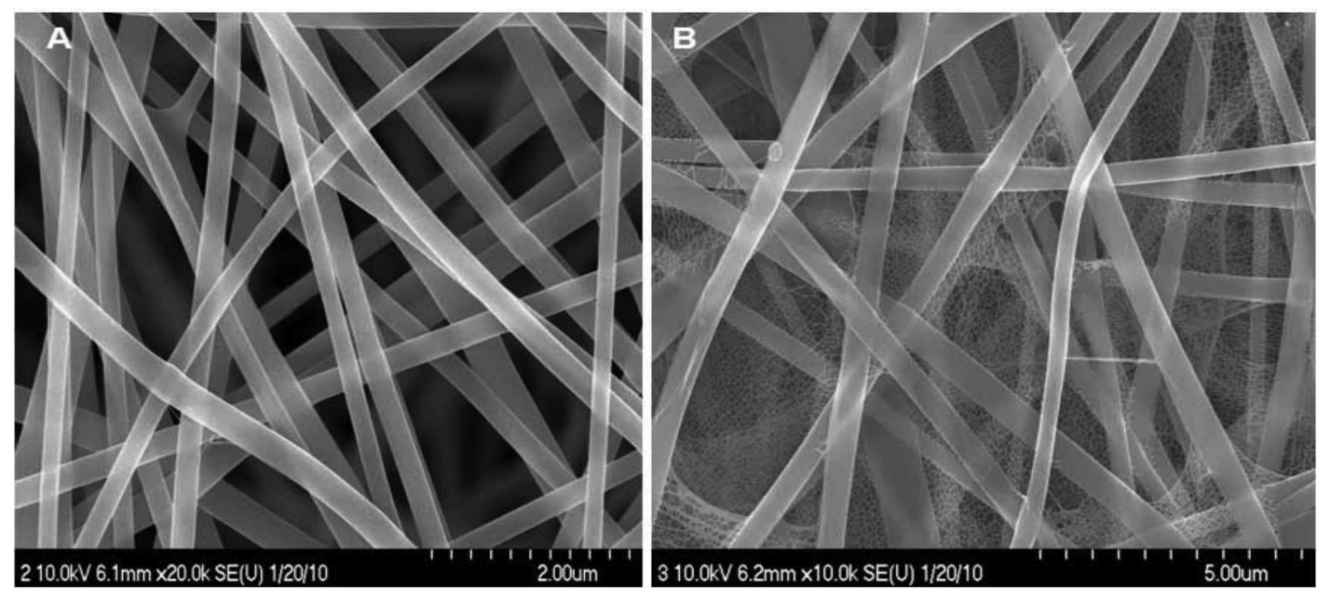

Fig. 4. FE-SEM images of (A) pure nylon-6 and (B) MPEGN (1 wt $\%$ MPEG) mats. 
Pant 113

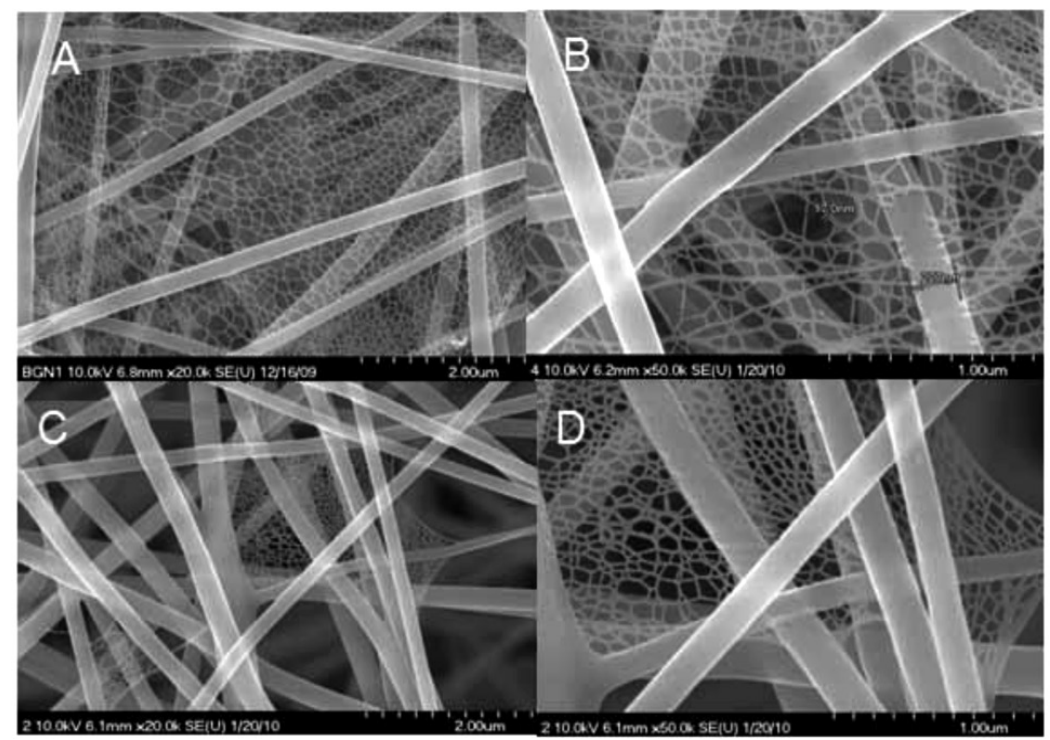

Fig. 5. FE-SEM images of MPEGN mats containing (A and $B$ ) $2 \mathrm{wt} \%$ and $(\mathrm{C}$ and $\mathrm{D}) 4 \mathrm{wt} \% \mathrm{MPEG}$ in MPEGN nanocomposite.

The mechanical strength of the nylon-6 nanofibers mat with low MPEG content was greater than that of pure nylon-6 and again decreased with increasing MPEG content (Fig. 7 A). The enhanced mechanical strength up to $1 \mathrm{wt} \%$ MPEG is due to the highly interconnected spider-web structure of MPEG with the larger nylon-6 backbone nanofibers. A small amount (up to 1\%) of MPEG in the nylon- 6 solution can give a high dispersion, and this promotes effective cross linking between two types of fibers to form spider-wave like structure during electrospinning. Upon increasing the MPEG content, the distribution of spider-web like structures decreased due to low dispersion and they occupied less space in the mat (not distributed throughout the mat), as can be observed in the FE-SEM image (Fig. 5). Therefore, there was a decrease in mechanical strength. These two different polymeric nanofibers may be interconnected by means of hydrogen bonds.

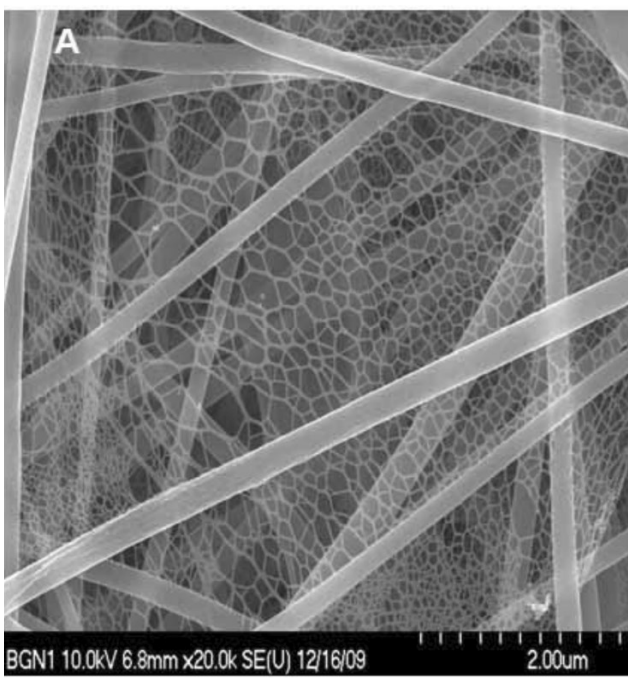

Fig. 6. FESEM images of MPEGN mat (a) before and (b) after water extraction.

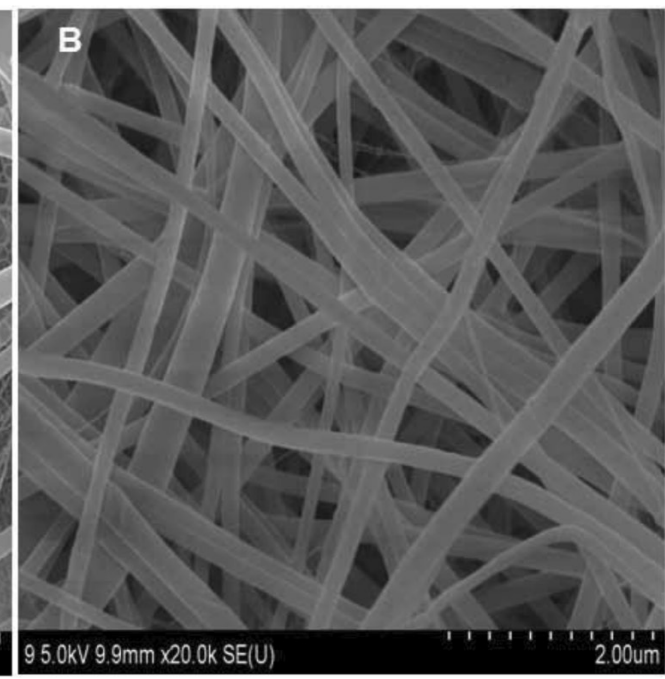




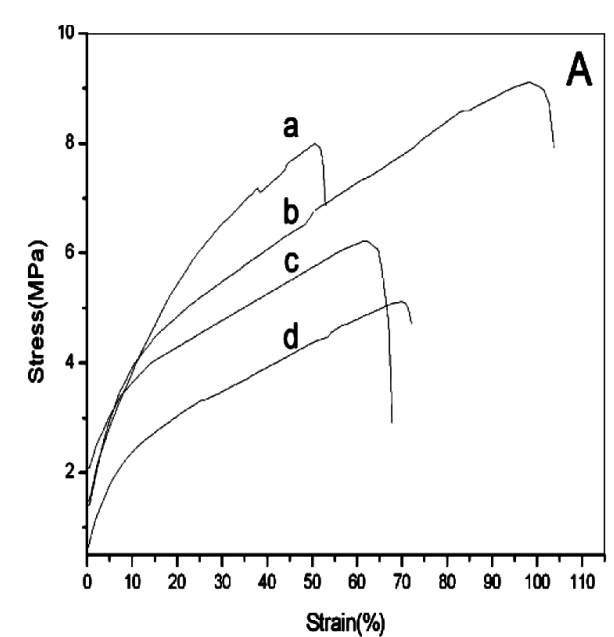

Fig. 7. (A) Mechanical strength

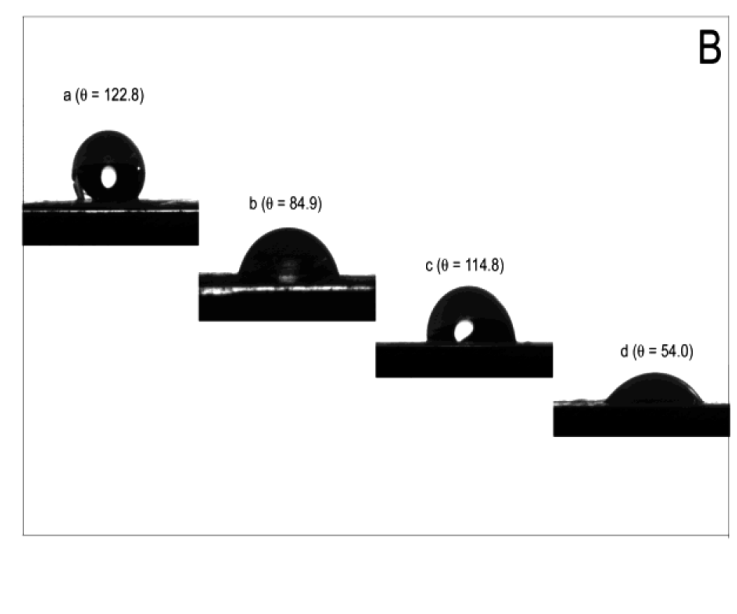

(B) contact angle of (a) 0 , (b) 1, (c) 2, and (d) $4 \mathrm{wt} \%$ MPEG containing nylon-6 mat.

Fig. 7B shows the water contact angle of the mat after $1 \mathrm{~s}$ for pure nylon-6 (a), $1 \mathrm{wt} . \%$ (b), $2 \mathrm{wt} . \%$ (c) and $4 \mathrm{wt} \%$ (d) MPEG in nylon-6 solution. It shows that a $1 \mathrm{wt} . \%$ MPEGN mat is not only much more hydrophilic than pure nylon-6 mat but also more hydrophilic than a $2 \mathrm{wt} \%$ MPEGN mat. It is probably due to the formation of more surface to volume ratio of well distributed sub nanofibers of hydrophilic MPEG throughout the $1 \mathrm{wt} \%$ MPEGN mat than the others (Fig. 4B). Such hydrophilic property of filter surface can effectevely absorbed air moister and microbioorganism during filtration.

\section{Conclusion}

MPEG oligomer, which was not fabricated by electrospinning alone, can be fabricated with viscous nylon-6 solution in the form of spider-web like nanofibers. Strongly interconnected thin MPEG spider-web like nanofibers with thick nylon-6 nanofibers, are responsible to increase the mechanical strength and hydrophilic nature of nylon- 6 mat. This high aspect ratio MPEGN nanofiber mat can be used for the effective air filtration and different biomedical application.

\section{References}

[1] Sang YM, Gu QB, Sun TC, Li FS and Liang CZ (2008), Journal of Hazardous Materials 153: 860.

[2] Yun KM, Hogan CJ, Matsubayashi Y, Kawabe M, Iskandar F and Okuyama K (2007) Chemical Engineering Science 62: 4751.

[3] Nova CJM, Paolucci-Jeanjean D, M.P. Belleville, M. Barboiu, M. Rivallin and G. Rios (2008) Journal of Membrane Science 321:81.

[4] Vitchuli N , Shi Q, Nowak J , McCord M , Bourham M , Zhang X. (2010) Journal of Applied Polymer

Science 116:2181.

[5] Kumbar SG, Nukavarapu SP, James R, Nair LS, Laurencin CT (2008) Biomaterials 29:4100. 
[6] Zhang YZ, Venugopal JR, El-Turki A, Ramakrishna S, Su B and Lim CT (2008), Biomaterials 29: 4314.

[7] Park SH, Kim TG, Kim HC, Yang DY and Park TG (2008), Acta Biomater 4: 1198.

[8] Raghavan P, Zhao XH, Kim JK, Manuel J, Chauhan GS, Ahn JH and Nan C (2008), Electrochemica Acta 54:228.

[9] Guo B, Zhao SZ, Han GY and Zhang LW (2008), Electrochimica Acta 53:5174.

[10] Sill TJ and Recum HAV (2008), Biomaterials 29:1989.

[11] Ramakrishna S, Fujihara K, Teo WE, Yong T, MaZW, Ramaseshan R (2006), Materials Today 9:40.

[12] Ding B, Chunrong L, Yasuhiro M, Kuwaki O, Shiratori S (2006), Nanotechnology 17:3685.

[13] Parajuli DC, Bajgai MP, Ko JA, Kang HK, Khil MS and Kim HY (2009), Applied Materials and Interfaces 1(4):750.

[14] Barakat NAM, Kanjwal MA, Sheikh FA and Kim HY (2009), Polymer 50: 4389.

[15] Heimann AC and Jakobsen R (2007) Talanta 72:839

[16] Aussawasathien D, Teerawattananon C and Vongachariy A (2008), Journal of Membrane Science 315:11.

[17] Kim C, Park SH, Lee WJ and Yang KS (2004), Electrochimica Acta 50:877.

[18] Heikkila P, Taipale A, Lehtimakl M and Harlin A (2008), Polymer Engineering Science 48:1169.

[19] Taylor, G. Proc. R. Soc. London 1969; A313: 1515.

[20] Yu JH.; Fridrikh SV and Rutledge GC (2006), Polymer 47:4789. 